\title{
Prediction of pathological and oncological outcomes based on extended prostate biopsy results in patients with prostate cancer receiving radical prostatectomy: a single institution study
}

Fumio Ishizaki ${ }^{1,3}$, Noboru Hara ${ }^{3 *}$, Hiroshi Koike ${ }^{1}$, Makoto Kawaguchi², Akira Tadokoro 1,3, Itsuhiro Takizawa ${ }^{3}$, Tsutomu Nishiyama ${ }^{3}$, Kota Takahashi ${ }^{3}$ and Rudolf Hohenfellner ${ }^{4}$

\begin{abstract}
Background: The prediction of pathological outcomes prior to surgery remains a challenging problem for the appropriate surgical indication of prostate cancer. This study was performed to identify preoperative values predictive of pathological and oncological outcomes based on standardized extended prostate biopsies with core histological results diagrammed/mapped in patients receiving radical prostatectomy for prostate cancer clinically diagnosed as localized or locally advanced disease.

Methods: In 124 patients with clinically localized or locally advanced prostate cancer (CT1c-cT3a) without prior treatment, pathological outcomes on the surgical specimen including seminal vesicle involvement (SVI), positive surgical margin (PSM), and perineural invasion (PNI) were studied in comparison with clinical parameters based on the results of 14-core prostate biopsies comprising sextant, laterally-directed sextant, and bilateral transition zone (TZ) sampling.

Results: Concerning the association of pathological outcomes with oncological outcomes, patients with PSM and PNI on surgical specimens had poorer biochemical-progression-free survival than those without PSM (logrank $p=0.002$ ) and PNI $(p=0.003)$; it was also poorer concerning SVI, although the difference was not significant $(p=0.120)$. Concerning the impact of clinical parameters on these pathological outcomes, positive TZ and multiple positive biopsy cores in the prostatic middle were independent values predictive of SVI with multivariate analyses ( $p=0.020$ and $p=0.025$, respectively); both positive TZ and multiple positive prostatic middle biopsies were associated with larger tumor volume ( $p<0.001$ in both). The percentage of positive biopsy cores (\%positive cores) and biopsy Gleason score were independent values predictive of PSM $(p=0.001)$ and PNI $(p=0.001)$, respectively. Multiple positive cores in the prostatic base were associated with proximal/bladder-side PSM $(p<0.001)$, and also linked to poorer biochemical-progression-free survival $(p=0.004)$. Clinical T stage had no association with these pathological outcomes.
\end{abstract}

Conclusions: \%positive cores and Gleason score in extended biopsies were independent values predictive of PSM and PNI in prostate cancer clinically diagnosed as localized or locally advanced disease, respectively, which were associated with poorer oncological outcomes. When diagramming biopsy-core results, extended biopsy may provide additional information for predicting oncological and pathological outcomes including SVI in patients clinically diagnosed as having localized or locally advanced disease.

Virtual slides: The virtual slide(s) for this article can be found here: http://www.diagnosticpathology.diagnomx.eu/vs/ 8790262771042628

\footnotetext{
* Correspondence: harasho@med.niigata-u.ac.jp

${ }^{3}$ Division of Urology, Department of Regenerative and Transplant Medicine, Graduate School of Medical and Dental Sciences, Niigata University, Nigata, Japan

Full list of author information is available at the end of the article
} 


\section{Background}

Since the advent of the preoperative staging table/nomogram, the clinical staging of localized or locally advanced prostate cancer has been revolutionized both for radical surgery and radiotherapy [1-3]. These predictive scales are used for the calculation of possible oncological outcomes or probability of extraprostatic extension in the pathological stage, for example, seminal vesicle involvement (SVI), by combining clinical parameters/variables such as serum variable prostate-specific antigen levels, digital rectal examination findings, Gleason score at prostate biopsy, and percentage of positive biopsy cores (\%positive cores) [1-3]. Although extraprostatic disease does not necessarily indicate incurable disease, SVI has been associated with poor oncological outcomes [3-6]. However, advances in the aforementioned staging modalities have led to the low prevalence of SVI in pathological outcomes, and it has been reported that the recent low prevalence of SVI makes the validation of prognostic models difficult [7]. On the other hand, positive surgical margin (PSM) and perineural invasion (PNI) have also been suggested to have a prognostic value $[6,8]$, but clinical parameters predictive of PSM remain controversial and studies on PNI are limited. The prediction of these pathological outcomes prior to surgery is significant in clinical practice, but there has been no study examining which clinical parameters reflect each of SVI, PSM, and PNI; such analyses may confirm the feasibility of staging nomograms also based on pathological approaches.

Several previous studies assessed pathological outcomes such as SVI in comparison with observations using conventional sextant to octant biopsies $[9,10]$, but there has been no study examining predictive values for the mentioned pathological outcomes based on extended/extensive prostate biopsies that may potentially provide more accurate information concerning the disease condition and extension [11]. The aim of the present study was to identify preoperative clinicopathological factors associated with SVI, PSM, and PNI, based on standardized extended prostate biopsies with core histological results precisely diagrammed in men who underwent radical prostatectomy for prostate cancer clinically diagnosed as localized or locally advanced disease. In addition, oncological outcomes were assessed to clarify whether such factors identified based on extended biopsy results also have a prognostic value in them.

\section{Methods}

\section{Patients}

We reviewed 144 consecutive patients, who were diagnosed with prostate cancer based on standardized 14core prostate biopsy described elsewhere and treated with standardized radical prostatectomy with standard or modified pelvic lymphadenectomy for localized or locally advanced prostate cancer (cT1c-3a N0 M0) at our institution between June 2003 and March 2010. Twenty patients who received prior therapy such as androgen deprivation or radiotherapy were excluded. The final study group comprised 124 patients. All surgical procedures were performed by an experienced urologist (HK) with assistance by experienced urologists ( $\mathrm{NH}$ and $\mathrm{AT}$ ). The procedure for this research project was approved by the Ethics Committee of our institution. Informed consent was obtained from all patients.

Clinical stages were determined according to the International Union Against Cancer (UICC) classification of 2009. Clinical staging routinely included abdominal and pelvic computerized tomography $(\mathrm{CT})$, chest radiograph or thoracic CT, isotope bone scanning, and extended/extensive prostate biopsy, as described elsewhere. Patients' demographics are shown in Table 1. PSA levels at diagnosis ranged between 2.6 and 63.7 (mean: $9.0 \mathrm{ng} / \mathrm{ml}$ ), and Gleason score at biopsy ranged between 6 and 10. Biochemical progression/PSA failure after surgery was defined as 2 consecutive PSA values of $0.2 \mathrm{ng} / \mathrm{ml}$ or greater at any time postoperatively, or any additional treatment more than 6 months after radical prostatectomy [12]. Five patients receiving adjuvant androgen deprivation therapy immediately after surgery were excluded from survival analyses. The mean and median observation periods were 31.0 and 24.0 months, respectively.

\section{Prostate biopsy}

Transrectal 14-core systemic prostate biopsies were carried out using transrectal ultrasound Aloka SSD 3300 CV (Aloka, Tokyo, Japan) following the method of standard sextant biopsy, in addition to those for sextant

Table 1 Patients' characteristics $(n=124)$

\begin{tabular}{ll}
\hline Age at diagnosis, mean (range) & $\mathbf{6 3 . 8}(\mathbf{4 0} \mathbf{- 7 3})$ \\
\hline PSA levels at diagnosis $(\mathrm{ng} / \mathrm{ml})$ & No. of patients \\
$<4.0$ & $13(10.5 \%)$ \\
$4.0-10.0$ & $75(60.5 \%)$ \\
$10.1-20.0$ & $29(23.4 \%)$ \\
$20.1-50.0$ & $6(4.8 \%)$ \\
50.1 or higher & $1(0.8 \%)$ \\
Gleason score at biopsy & No. of patients \\
6 & $48(38.7 \%)$ \\
$3+4$ & $20(16.1 \%)$ \\
$4+3$ & $20(16.1 \%)$ \\
8 or higher & $36(29.0 \%)$ \\
\hline
\end{tabular}

PSA, prostate-specific antigen. 
laterally directed biopsy and bilateral single core transition zone biopsy, as described previously [13]; 14 cores thus comprised 4 cores for each of the prostatic base, middle, and apex, and 2 cores for the transition zone. We separated the patients to subgroups based on those with a single positive core or multiple positive cores in each of the prostatic apex, middle, and base; this dichotomization was determined based on previous studies reporting that a single positive core in sextant or less biopsies is a requirement for categorizing to low-risk disease [14]. The maximal core length obtained by needle biopsy was $22 \mathrm{~mm}$; the mean measured core length was $15.9 \pm 3.2 \mathrm{~mm}$. Each core was tagged and mapped to produce histological localization diagrams (Figure 1).

\section{Histopathological diagnosis}

An independent pathologist (MK) made all histopathological diagnoses, and standardized grading was carried out according to the contemporary Gleason classification system [15]. All pathological examinations were performed using the whole-mount step-section technique. The prostate was weighed, measured, and inked, and was subsequently fixed in buffered formalin for at least 24 hours before sectioning. Following fixation, the specimen was step-sectioned, and was submitted in entire cross-sections from the apex to base at $3-5-\mathrm{mm}$ intervals; the mean number of sections was $27.1 \pm 6.6$. The apex and bladder neck were coned and serially sectioned. Cancer foci were also diagrammed in photographs of the sectioned specimen. SVI was defined following the criteria of Epstein et al., as the invasion to the muscular wall of the seminal vesicle [16]. PNI in the radical prostatectomy specimens was assessed following the method reported previously [17-19]. Surgical margins were assessed adopting an approach similar to that in the recent consensus criteria [20].

\section{Statistical analysis}

In addition to the chi-square and Fisher's exact tests, the Mann-Whitney $U$ test was used to compare unpaired parameters between two subgroups. Independent predictive values were identified with multivariate analysis using a logistic regression model; variables having $\mathrm{p}$-value of $\mathrm{p}<0.1$ in univariate analyses were tested with multivariate analysis. Statistical analysis of Gleason score was performed with a stepwise model. Survival curves were generated using the method of Kaplan and Meier, and differences between curves were evaluated employing the log-rank test. Uniand multivariate analyses for survival-associated parameters were performed using Cox proportional hazard models. Correlations between parameters were analyzed using Spearman's rank correlation coefficient (rs) analysis. The test was two-sided except for the Fisher's exact test, and $\mathrm{p}$ $<0.05$ was considered significant. All analyses were performed using SPSS version 15.0 J (SPSS Inc., Chicago, IL, USA) on a Windows-based computer.

\section{Results}

Pathological stage and Gleason score in radical prostatectomy specimens

There were 81 (65.3\%) with pT2 and $42(33.9 \%)$ patients with pT3 disease. Thirty-six (29.3\%) and 4 (3.2\%) of the patients experienced pathological upstaging from and downstaging, respectively. One patient had pT0 disease. Also, there were 48 (38.7\%) with pathological Gleason score of 6, 40 (32.3\%) with pathological Gleason score of 7 , and $36(29.0 \%)$ patients with pathological Gleason score of 8 or higher. Forty-eight (39.0\%) and 27 (22.0\%) of the patients experienced upgrade and downgrade of Gleason score, respectively.

\section{Relationship between SVI and clinical parameters or biopsy findings}

Table 2 shows the relationships between the presence/ absence of SVI and clinical parameters or tumor localizations based on prostate biopsies. PSA levels, \%positive cores, and the cT stage were not different between those with and without SVI. Patients with SVI showed a higher Gleason score at biopsy, higher rate of multiple (2 or more) positive cores in the prostatic middle, and a higher transition zone-positive rate, compared with those without SVI $(\mathrm{p}=0.005, \mathrm{p}=0.005$, and $\mathrm{p}=0.002$,

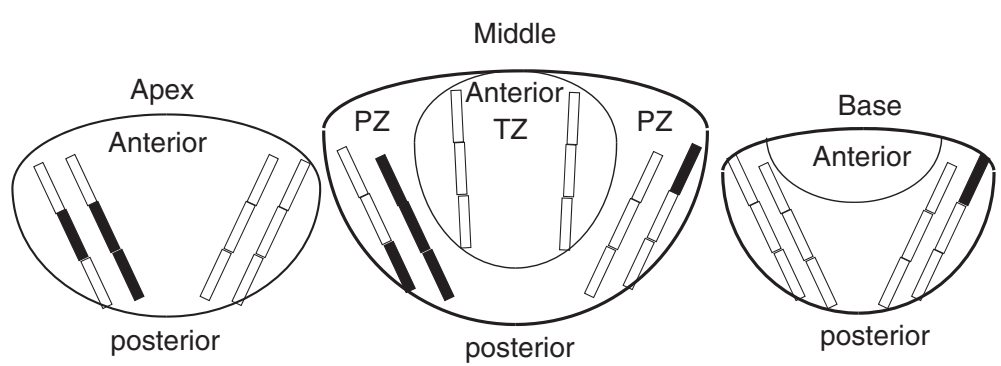

Figure 1 Histological localization diagram in a 67 year-old man with 6 positive cores in 14-core prostate biopsy, showing positive segments in cores as bars painted in black (TZ, transition zone; PZ, peripheral zone); each core was histologically assessed in 3 divided core segments. 
Table 2 Variance in preoperative clinicopathological parameters between patients with and without seminal vesicle involvement (SVI)

\begin{tabular}{|c|c|c|c|c|c|}
\hline & \multirow{2}{*}{$\begin{array}{c}\text { SVI } \\
\text { present } \\
(\mathrm{n}=8)\end{array}$} & \multirow{2}{*}{$\begin{array}{c}\text { SVI } \\
\text { absent } \\
(n=116)\end{array}$} & \multirow{2}{*}{$\frac{\text { Univariate }}{P \text { value }}$} & \multicolumn{2}{|r|}{ Multivariate } \\
\hline & & & & $P$ value & Adjusted odds ratio $(95 \% \mathrm{Cl})$ \\
\hline Mean age [years] & $63.6 \pm 3.9$ & $63.9 \pm 5.1$ & 0.725 & & \\
\hline PSA [ng/ml] & $13.0 \pm 8.1$ & $8.7 \pm 7.2$ & 0.105 & & \\
\hline Gleason score at biopsy & $8.1 \pm 1.1$ & $7.0 \pm 1.0$ & 0.005 & & \\
\hline CT stage $(n)$ & & & 0.153 & & \\
\hline T1c & $5(62.5 \%)$ & $94(81.0 \%)$ & & & \\
\hline $\mathrm{T} 2$ & $3(37.5 \%)$ & $15(12.9 \%)$ & & & \\
\hline T3a & $0(0 \%)$ & $7(6.0 \%)$ & & & \\
\hline \%positive cores & $31.0 \pm 21.3$ & $21.3 \pm 16.4$ & 0.242 & & \\
\hline TZ biopsy positive (n) & $7(87.5 \%)$ & $35(30.2 \%)$ & 0.002 & 0.023 & $12.99(1.490-111.1)$ \\
\hline Number of apex-positive (2-4/0-1) (n) & $3 / 5$ & $26 / 90$ & 0.278 & & \\
\hline Number of middle-positive $(2-4 / 0-1)(n)$ & $6 / 2$ & $28 / 88$ & 0.005 & 0.025 & $7.299(1.312-40.00)$ \\
\hline Number of base-positive $(2-4 / 0-1)(n)$ & $4 / 4$ & $23 / 93$ & 0.067 & & \\
\hline
\end{tabular}

PSA, prostate-specific antigen; TZ, transition zone; apex, prostatic apex biopsy; middle, prostatic middle biopsy; base, prostatic base biopsy.

respectively); with multivariate analyses using the logistic regression model, positive transition zone (adjusted odds ratio: 12.99 [95\%CI: $1.490-111.1$ ], $\mathrm{p}=0.023$ ) and multiple positive biopsies in the prostatic middle (adjusted odds ratio: 7.299 [95\% CI: $1.312-40.00], \mathrm{p}=0.025)$ were independent values predictive of seminal vesicle involvement.

Results when analyzing data on each of the standard sextant sites or laterally-directed sextant cores had no association with pathological outcomes (data not shown). When assessed with tumor localization in radical prostatectomy specimens, the positive-predictive value of TZ biopsy was 0.86 ; $86 \%$ of the patients with positive $\mathrm{TZ}$ biopsy also had $\mathrm{TZ}$ cancer or cancer invading to TZ in surgical specimens. All of the patients with positive prostatic middle biopsy also had cancer foci in the prostatic middle in surgical specimens. The largest tumor size ranged between $2-45$ (mean: $16.8 \pm 9.4$ ) mm (pT0 in one patient) in prostatectomy specimens; patients with a positive $\mathrm{TZ}$ biopsy had a larger tumor size than those with a negative TZ biopsy (mean $21.5 \pm 10.2 \mathrm{~mm} v s$. $14.2 \pm 7.8 \mathrm{~mm}$, respectively, $\mathrm{P}<0.001)$. Also, patients with multiple positive cores in prostatic middle biopsy had a larger tumor size than those with no or a single positive core in prostatic middle biopsy (mean: $22.0 \pm 11.3 \mathrm{~mm} v s$. $14.9 \pm 7.7 \mathrm{~mm}$, respectively, $\mathrm{P}<0.001)$.

\section{Relationship between PSM and clinical parameters or biopsy findings}

Table 3 shows the relationships between the presence/ absence of PSM and clinical parameters or tumor localizations based on prostate biopsies. Data on the surgical margin were not available in 4 patients. PSA levels, biopsy Gleason score, and cT stage were not different between those with and without PSM. Patients with PSM showed higher \%positive cores and a higher rate of multiple positive cores in the prostatic base, middle, and apex, compared with those without PSM ( $\mathrm{p}=0.001$, $0.049,0.004$, and 0.037 , respectively); with multivariate analyses using the logistic regression model, \%positive cores was an independent value predictive of PSM (adjusted odds ratio: 1.044 [95\%CI: 1.019-1.070], $\mathrm{p}=0.001$, Table 4). \%positive cores was also correlated with the largest tumor size in prostatectomy specimens (rs $=0.354, \mathrm{p}<0.001)$.

\section{Relationship between PNI and clinical parameters or biopsy findings}

Table 4 shows the relationships between the presence/ absence of PNI and clinical parameters or tumor localizations based on prostate biopsies. Gleason score on biopsy alone was higher in patients with than in those without PNI (univariate $\mathrm{p}<0.001$, multivariate $\mathrm{p}=0.001$, adjusted odds ratio: 1.983 [95\%CI: 1.342-2.929]).

\section{Oncological outcomes}

Five men receiving immediate adjuvant androgen deprivation therapy and those with missing data were excluded from survival analyses to assess appropriate biochemical progression. Regarding pathological outcomes, patients with PSM had poorer biochemicalprogression-free survival than those without PSM (logrank $\mathrm{p}=0.002)$, and this was also the case for PNI $(\mathrm{p}=0.003) \quad$ (Figure 2). Although patients with SVI seemingly showed a lower biochemical-progression-free survival rate than those without SVI, the difference 
Table 3 Variance in preoperative clinicopathological parameters between patients with and without positive surgical margin (PSM)

\begin{tabular}{|c|c|c|c|c|c|}
\hline & \multirow{2}{*}{$\begin{array}{c}\text { PSM } \\
\text { present } \\
(\mathrm{n}=39)\end{array}$} & \multirow{2}{*}{$\begin{array}{c}\text { PSM } \\
\text { absent } \\
(n=81)\end{array}$} & \multirow{2}{*}{$\frac{\text { Uniivariate }}{\mathrm{p} \text {-value }}$} & \multicolumn{2}{|r|}{ Multivariate } \\
\hline & & & & $P$ value & Adjusted odds ratio $(95 \% \mathrm{Cl})$ \\
\hline Mean age [years] & $63.5 \pm 36.0$ & $64.0 \pm 4.6$ & 0.993 & & \\
\hline PSA [ng/ml] & $9.8 \pm 6.3$ & $8.7 \pm 7.8$ & 0.247 & & \\
\hline Gleason score at biopsy & $7.2 \pm 1.2$ & $7.0 \pm 1.0$ & 0.209 & & \\
\hline cT stage $(n)$ & & & 0.347 & & \\
\hline $\mathrm{T} 1 \mathrm{c}$ & $30(76.9 \%)$ & $65(80.2 \%)$ & & & \\
\hline $\mathrm{T} 2$ & $5(12.8 \%)$ & $13(16.0 \%)$ & & & \\
\hline T3a & $4(10.3 \%)$ & $3(3.7 \%)$ & & & \\
\hline \%positive cores & $31.0 \pm 21.3$ & $21.3 \pm 16.4$ & 0.001 & 0.001 & $1.044(1.019-1.070)$ \\
\hline TZ biopsy positive (n) & $17(43.6 \%)$ & $24(29.6 \%)$ & 0.131 & & \\
\hline Number of apex-positive $(2-4 / 0-1)(n)$ & $14 / 25$ & $15 / 66$ & 0.037 & & \\
\hline Number of middle-positive $(2-4 / 0-1)(n)$ & $17 / 22$ & $15 / 66$ & 0.004 & & \\
\hline Number of base-positive (2-4/0-1) (n) & $13 / 26$ & $14 / 67$ & 0.049 & & \\
\hline
\end{tabular}

PSA, prostate-specific antigen; TZ, transition zone; apex, prostatic apex biopsy; middle, prostatic middle biopsy; base, prostatic base biopsy. Data not available in 4 patients.

between survival curves were not significant (logrank $\mathrm{p}=0.120$ ) (Figure 2). Among preoperative clinical parameters, patients with PSA levels of $10 \mathrm{ng} / \mathrm{ml}$ or higher had poorer biochemical-progression-free survival than those with PSA levels less than $10 \mathrm{ng} / \mathrm{ml} \quad(\mathrm{p}=0.033)$. Biochemical-progression-free survival was lower in patients with biopsy Gleason score of 8 or higher than in those with Gleason score of $<=7(p=0.027)$. Patients with $\%$ positive cores of $>=35.7 \%$ (number of positive cores of 5 or more) showed poorer biochemical-progression-free survival than those with \%positive cores of $<35.7 \%$ $(\mathrm{p}=0.023)$. Patients with multiple positive cores in the prostatic base had poorer biochemical-progression-free survival ( $\mathrm{p}=0.004)$; patients with multiple positive cores in the prostatic base more frequently had proximal/bladderside PSM than those with a single or no positive core in the prostatic base (48.1 vs. $6.5 \%$, respectively, $\mathrm{p}<0.001$ ). We additionally showed biochemical-progression-free survivals according to pT stage (Figure 3 ).

\section{Discussion}

In the present study, PSM was a significant pathological factor associated with poorer oncological outcomes, and \%positive cores was an independent value predictive of PSM (multivariate $\mathrm{p}=0.001$ ) showing a prognostic significance $(p=0.023)$ in patients with prostate cancer

Table 4 Variance in preoperative clinicopathological parameters between patients with and without peruneural invasion (PNI)

\begin{tabular}{|c|c|c|c|c|c|}
\hline & \multirow{2}{*}{$\begin{array}{c}\text { PNI } \\
\text { present } \\
(n=63)\end{array}$} & \multirow{2}{*}{$\begin{array}{c}\text { PNI } \\
\text { absent } \\
(n=61)\end{array}$} & \multirow{2}{*}{$\frac{\text { Univariate }}{P \text { value }}$} & \multicolumn{2}{|r|}{ Multivariate } \\
\hline & & & & $P$ value & Adjusted odds ratio $(95 \% \mathrm{Cl})$ \\
\hline Mean age [years] & $63.7 \pm 5.0$ & $64.0 \pm 5.0$ & 0.894 & & \\
\hline PSA [ng/ml] & $9.5 \pm 5.9$ & $8.5 \pm 8.5$ & 0.104 & & \\
\hline Gleason score at biopsy & $7.4 \pm 1.1$ & $6.7 \pm 0.9$ & $<0.001$ & 0.001 & $1.983(1.342-2.929)$ \\
\hline CT stage (n) & & & 0.615 & & \\
\hline T1c & $49(77.8 \%)$ & $50(82.0 \%)$ & & & \\
\hline $\mathrm{T} 2$ & $9(14.3 \%)$ & $9(14.8 \%)$ & & & \\
\hline T3a & $5(7.9 \%)$ & $2(3.3 \%)$ & & & \\
\hline \%positive cores & $23.5 \pm 18.7$ & $19.8 \pm 14.8$ & 0.513 & & \\
\hline TZ biopsy positive (n) & $24(38.1 \%)$ & $18(29.5 \%)$ & 0.312 & & \\
\hline Number of apex-positive (2-4/0-1) (n) & $14 / 49$ & $15 / 46$ & 0.755 & & \\
\hline Number of middle-positive (2-4/0-1) (n) & $21 / 42$ & $13 / 48$ & 0.134 & & \\
\hline Number of base-positive $(2-4 / 0-1)(n)$ & $17 / 46$ & $10 / 51$ & 0.153 & & \\
\hline
\end{tabular}

PSA, prostate-specific antigen; TZ, transition zone; apex, prostatic apex biopsy; middle, prostatic middle biopsy; base, prostatic base biopsy. 

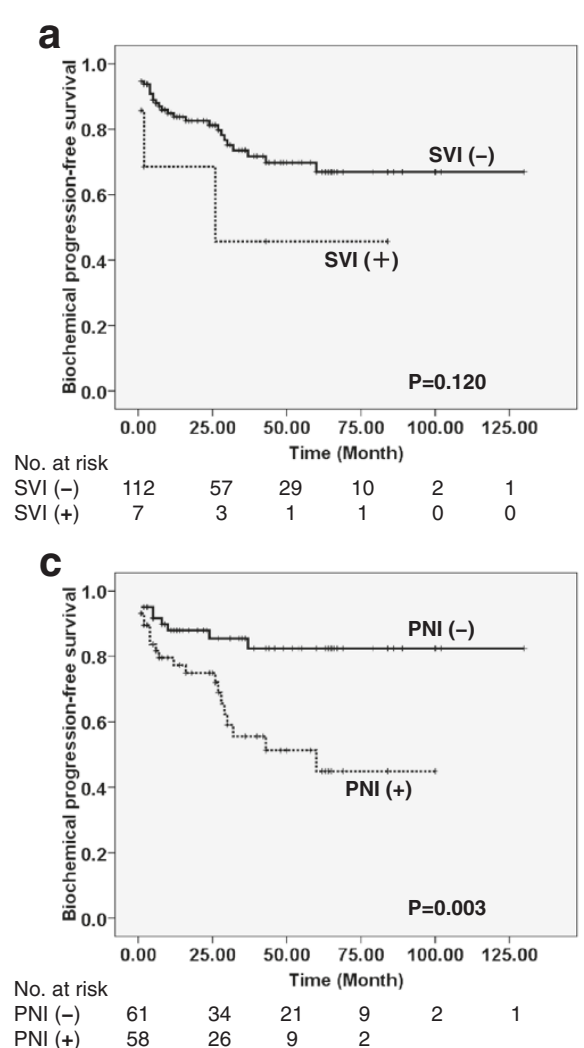

b

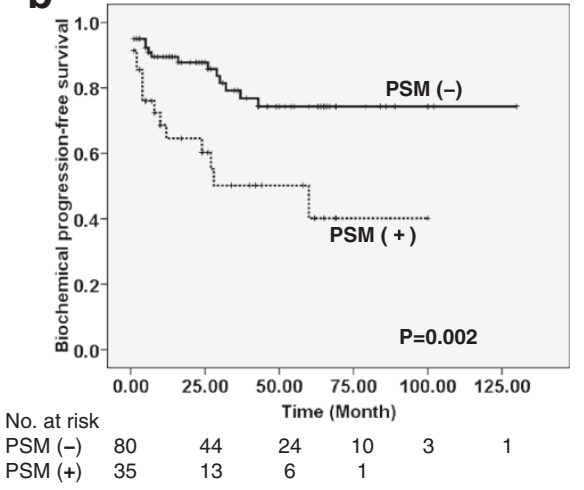

Figure 2 Biochemical-progression-free survival in patients with or without seminal vesicle involvement (SVI, A), a positive surgical margin (PSM, B), and perineural invasion (PNI, C).

clinically diagnosed as localized or locally advanced disease. These results suggest that \%positive cores based on extended biopsies may be useful for predicting oncological outcomes. \%positive cores theoretically reflects the tumor volume [21], and thus is a feasible index for predicting PSM. In addition, patients with multiple positive cores in the prostatic base had poorer oncological outcomes, and they more frequently had proximal/bladder-side PSM than those with a single or no positive core in the prostatic base (48.1 vs. $6.5 \%$, respectively, $\mathrm{p}$ $<0.001)$. Several previous studies have reported that proximal-side PSM is an adverse prognostic factor [22], and it has also been shown that positive cores in the prostatic base are associated with a higher rate of proximal PSM [23], but the relevant previous studies did not perform survival analyses. The present study additionally suggested that multiple positive cores in the prostatic base with extended biopsy protocol possibly have a prognostic significance, although further studies are required to draw a conclusion.

Previous studies have reported the frequency of PNI in radical prostatectomy specimens to be between 32 and 79\%. [19-21], and it was about $50 \%$ in the present study (Table 3). A few studies reported that the presence of
PNI is associated with poor oncological outcomes [8], and our study also supported the prognostic value of PNI. The current study also suggested that biopsy Gleason score was an independent value predictive of PNI. It has been shown that PNI of prostate cancer is relevant to a tumor's invasive capacity [24], while Gleason score

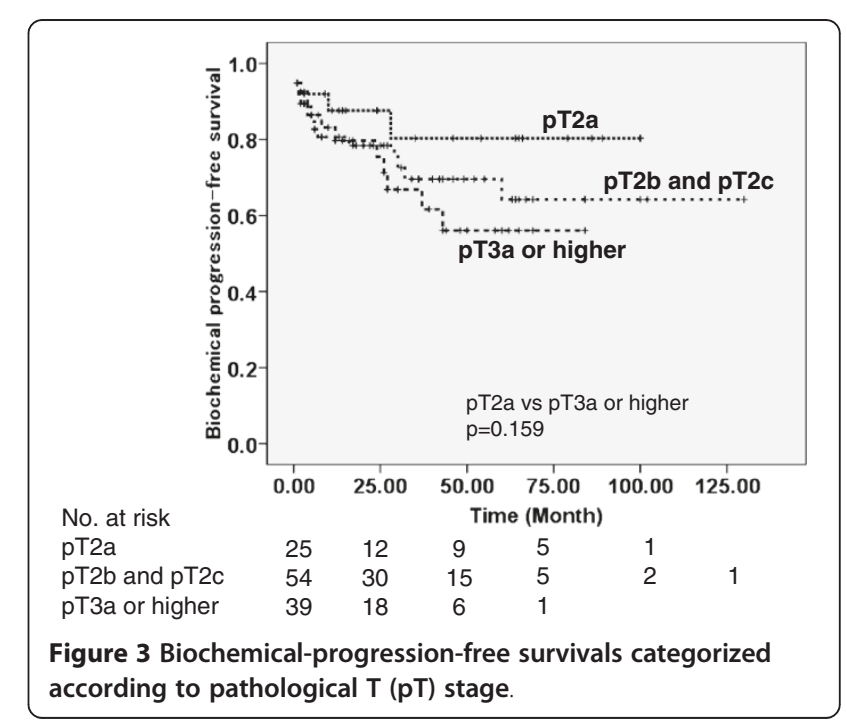


is also involved in invasiveness of prostate cancer in relation to the expression of matrix metalloproteinases $[25,26]$. A study regarding the diagnostic value of PNI in extended biopsy cores is currently underway.

Recent advances in staging modalities and surgical indications have been associated with the low prevalence of SVI in surgical outcomes, and it has been reported that the recent low prevalence of SVI makes the validation of prognostic models/nomogram difficult [7]. SVI did not have a prognostic significance in the present study most probably due to the small number of those with SVI; the survival curves categorized with and without SVI seemingly separated (Figure 2). Additionally, the 5 patients excluded from survival analyses because of immediate inception of adjuvant androgen deprivation therapy showed SVI, and their exclusion probably contributed to the absence of statistical significance for SVI. There has been no study examining associations between these pathological conditions and the results of precisely profiled extended biopsy. When mapping/diagramming positive cores, extended biopsy may be of value to predict SVI in patients with prostate cancer clinically diagnosed as localized disease. Interestingly, the number/percentage of positive cores calculated for the standard sextant sites or laterallydirected sextant cores did not reflect SVI, whereas positive $\mathrm{TZ}$ and multiple positive cores in the prostatic middle were independently associated with SVI. However, positive-predictive value of TZ biopsy assessed with tumor localization in the prostatectomy specimen was 0.86 , suggesting contaminations of cancer arising from the peripheral zone. Patients with a positive TZ biopsy or multiple positive cores in prostatic middle biopsy had a larger tumor size in prostatectomy specimens. With the current 14-core biopsy protocol, parameters such as positive $\mathrm{TZ}$ and multiple positive cores in the prostatic middle possibly reflect the tumor size, and thus are associated with SVI. To validate the significance of these observations, studies comparing various biopsy protocols are needed.

In the present study with the mentioned extended biopsy protocol, positive $\mathrm{TZ}$ and multiple positive cores in the prostatic middle were independently correlated with SVI ( $\mathrm{p}=0.020$ and $\mathrm{p}=0.023$, respectively). Also, $\%$ positive cores was an independent factor predictive of PSM ( $\mathrm{p}=0.001)$, and biopsy Gleason score was independently correlated with PNI $(\mathrm{p}=0.001)$. Thus, clinical factors predictive of pathological outcomes varied among SVI, PSM, and PNI; these results suggest that prevailing staging nomograms or risk-assessment scoring systems combining multiple values such as Gleason score and \%positive cores are practically possible based on the pathological approach employing extended biopsy protocols [1-3]. Additionally, artificial intelligence techniques such as artificial neural networks incorporating mentioned informations obtained from extended biopsies potentially support decision-making in prostate cancer treatment [27].

In this study, one patient $(0.8 \%)$ had pT0 disease, and the frequency was similar to those in previous reports [28]. The present study had several limitations. The study was retrospectively designed, and the relatively small number of patients may have led to a limited statistical power. Also, the small number of locally advanced disease cases is a limitation of this type of study; surgical indication probably affected the background of the study group, as discussed elsewhere. Further follow-up studies are warranted to confirm the significance of the observations on oncological outcomes in men with localized prostate cancer.

\section{Conclusions}

PSM and PNI were significant pathological factors associated with poorer oncological outcomes, and \%positive cores and Gleason score in extended biopsies were independent values predictive of PSM and PNI in patients with prostate cancer clinically diagnosed as localized disease, respectively. When mapping/diagramming positive cores, extended prostate biopsies may provide additional value in the prediction of oncological and pathological outcomes such as SVI; multiple positive cores in the prostatic base possibly lead to poorer oncological outcomes.

\section{Abbreviations}

CT: Computerized tomography; PSA: Prostate-specific antigen; TZ: Transition zone; SVI: Seminal vesicle involvement; PSM: Positive surgical margin;

PNI: Perineural invasion.

\section{Competing interests}

The authors declare that they have no competing interests.

\section{Acknowledgements}

The authors thank Mr. Yuji Fujinawa, Mr. Osamu Yamamoto, and Ms. Takako Matsushima for their expert technical assistance in histopathological

examinations. This work has not been funded by any commercial company. It was supported in part by a Grant-in-Aid-for Scientific Research from the Ministry of Education, Culture, Sports, Science and Technology, Japan (\#21791493).

\section{Author details}

${ }^{1}$ Department of Urology, Joetsu 942-8502, Japan. ${ }^{2}$ Pathology, Niigata Rousai Hospital, Joetsu 942-8502, Japan. ${ }^{3}$ Division of Urology, Department of Regenerative and Transplant Medicine, Graduate School of Medical and Dental Sciences, Niigata University, Nigata, Japan. ${ }^{4}$ Department of Urology, Johannes Gutenberg University, Mainz, Germany.

\section{Authors' contributions}

$\mathrm{FI}$ performed data analysis and helped to draft the manuscript. NH wrote the manuscript and supervised throughout the study. HK carried out all surgical procedures and participated in data collection. MK performed all histopathological diagnoses. AT assisted surgery, clinical management of patients, and data collection. IT and TN helped data analysis. KT assisted to draft the manuscript. RH was a supervisor of this study. All authors read and approved the final manuscript.

Received: 9 April 2012 Accepted: 14 June 2012

Published: 14 June 2012

\section{References}

1. Partin AW, Kattan MW, Subong EN, Walsh PC, Wojno KJ, Oesterling JE, Scardino PT, Pearson JD: Combination of prostate-specific antigen, clinical 
stage, and Gleason score to predict pathological stage of localized prostate cancer: a multi-institutional update. JAMA 1997, 277:1445-1451.

2. Blute ML, Bergstralh EJ, Partin AW, Walsh PC, Kattan MW, Scardino PT, Montie JE, Pearson JD, Slezak JM, Zincke H: Validation of Partin tables for predicting pathological stage of clinically localized prostate cancer. J Urol 2000, 164:1591-1595.

3. Kattan MW, Potters L, Blasko JC, Beyer DC, Fearn P, Cavanagh W, Leibel S, Scardino PT: Pretreatment nomogram for predicting freedom from recurrence after permanent prostate brachytherapy in prostate cancer. Urology 2001, 58:393-399.

4. Yamamoto S, Kawakami S, Yonese J, Fujii Y, Tsukamoto T, Ohkubo Y, Komai $Y$, Ishikawa Y, Fukui I: Prognostic significance of cancer volume involving seminal vesicles in patients with pT3bpN0 prostate cancer. Urology 2008, 72:1224-1228.

5. Swanson GP, Goldman B, Tangen CM, Chin J, Messing E, Canby-Hagino E, Forman JD, Thompson IM, Crawford ED: The prognostic impact of seminal vesicle involvement found at prostatectomy and the effects of adjuvant radiation: data from Southwest Oncology Group 8794. J Urol 2008, 180:2453-2458.

6. Hull GW, Rabbani F, Abbas F, Wheeler TM, Kattan MW, Scardino PT: Cancer control with radical prostatectomy alone in 1000 consecutive patients. Urol 2002, 167:528-534.

7. Augustin H, Sun M, Isbarn H, Pummer K, Karakiewicz P: Decision curve analysis to compare 3 versions of Partin Tables to predict final pathologic stage. Urol Oncol, doi:10.1016/j.urolonc.2010.07.003 [in press].

8. Jeon HG, Bae J, YI JS, Hwang IS, Lee E, Lee SE: Perineural invasion is a prognostic factor for biochemical failure after radical prostatectomy. Int J Urol 2009, 16:682-686.

9. Ohori M, Kattan MW, Yu C, Matsumoto K, Satoh T, Ishii J, Miyakawa A, Irie A, Iwamura $\mathrm{M}$, Tachibana M: Nomogram to predict seminal vesicle invasion using the status of cancer at the base of the prostate on systematic biopsy. Int J Urol 2010, 17:534-540.

10. Karakiewicz Pl, Hutterer GC: Predictive models and prostate cancer. Nat Clin Pract Urol 2008, 5:82-92.

11. Singh PB, Nicholson CM, Ragavan N, Blades RA, Martin FL, Matanhelia SS: Risk of prostate cancer after detection of isolated high-grade prostatic intraepithelial neoplasia (HGPIN) on extended core needle biopsy: a UK hospital experience. BMC Urol 2009, 9:3.

12. Cooperberg MR, Pasta DJ, Elkin EP, Litwin MS, Latini DM, Du Chane J, Carroll PR: The University of California, San Francisco Cancer of the Prostate Risk Assessment score: a straightforward and reliable preoperative predictor of disease recurrence after radical prostatectomy. J Urol 2005, 173:1938-1942.

13. Hara N, Okuizumi M, Koike H, Kawaguchi M, Bilim V: Dynamic contrastenhanced magnetic resonance imaging (DCE-MRI) is a useful modality for the precise detection and staging of early prostate cancer. Prostate 2005, 62:140-147.

14. Boorjian SA, Karnes RJ, Rangel LJ, Bergstralh EJ, Blute ML: Mayo Clinic validation of the D'amico risk group classification for predicting survival following radical prostatectomy. J Urol 2008, 179:1354-1360.

15. Egevad L, Mazzucchelli R, Montironi R: Implications of the international society of urological pathology modified Gleason grading system. Arch Pathol Lab Med 2012, 136:426-434.

16. Epstein $\mathrm{Jl}$, Carmichael M, Walsh PC: Adenocarcinoma of the prostate invading the seminal vesicle: definition and relation of tumor volume, grade and margins of resection to prognosis. J Urol 1993, 149:1040-1045.

17. Ozcan F: Correlation of perineural invasion on radical prostatectomy specimens with other pathologic prognostic factors and PSA failure. Eur Urol 2001, 40:308-312.

18. Endrizzi J, Seay T: The relationship between early biochemical failure and perineural invasion in pathological T2 prostate cancer. BJU Int 2000, 85:696-698.

19. Ng JC, Koch MO, Daggy JK, Cheng L: Perineural invasion in radical prostatectomy specimens: lack of prognostic significance. J Urol 2004, 172:2249-2251.

20. Tan PH, Cheng L, Srigley JR, Griffiths D, Humphrey PA, van der Kwast TH, Montironi R, Wheeler TM, Delahunt B, Egevad L, Epstein JI: ISUP Prostate Cancer Group: International Society of Urological Pathology (ISUP) Consensus Conference on Handling and Staging of Radical Prostatectomy Specimens. Working group 5: surgical margins. Mod Pathol 2011, 24:48-57.
21. Pe ML, Trabulsi EJ, Kedika R, Pequignot E, Dicker AP, Gomella LG, Valicenti RK: Effect of percentage of positive prostate biopsy cores on biochemical outcome in low-risk PCa treated with brachytherapy or 3DCRT. Urology 2009, 73:1328-1334.

22. Buschemeyer WC III, Hamilton RJ, Aronson WJ, Presti JC Jr, Terris MK, Kane $\mathrm{CJ}$, Amling CL, Freedland SJ: Is a positive bladder neck margin truly a T4 lesion in the prostate specific antigen era? Results from the SEARCH Database. J Urol 2008, 179:124-129.

23. Touma NJ, Chin JL, Bella T, Sener A, Izawa Jl: Location of a positive biopsy as a predictor of surgical margin status and extraprostatic disease in radical prostatectomy. BJU Int 2006, 97:259-262.

24. Li R, Wheeler T, Dai H, Ayala G: Neural cell adhesion molecule is upregulated in nerves with prostate cancer Invasion. Hum Pathol 2003, 34:457-461.

25. Weidner N, Carroll PR, Flax J, Blumenfeld W, Folkman J: Tumor angiogenesis correlates with metastasis in invasive prostate carcinoma. Am J Pathol 1993, 143:401-409.

26. Angelucci A, Pace G, Sanità P, Vicentini C, Bologna M: Tissue print of prostate biopsy: a novel tool in the diagnostic procedure of prostate cancer. Diagn Pathol 2011, 6:34

27. Rodolfo Montironi: Automated decision algorithms in prostate cancer. Diagn Pathol 2010, 5(Suppl 1):S16.

28. Mazzucchelli R, Barbisan F, Tagliabracci A, Lopez-Beltran A, Cheng L, Scarpelli $M$, Montironi R: Search for residual prostate cancer on pT0 radical prostatectomy after positive biopsy. Virchows Arch 2007, 450:371-378.

doi:10.1186/1746-1596-7-68

Cite this article as: Ishizaki et al:: Prediction of pathological and oncological outcomes based on extended prostate biopsy results in patients with prostate cancer receiving radical prostatectomy: a single institution study. Diagnostic Pathology 2012 7:68.

\section{Submit your next manuscript to BioMed Central and take full advantage of:}

- Convenient online submission

- Thorough peer review

- No space constraints or color figure charges

- Immediate publication on acceptance

- Inclusion in PubMed, CAS, Scopus and Google Scholar

- Research which is freely available for redistribution 